\title{
A rare case report: Intrauterine diagnosis of fetal left ventricular aneurysm
}

\author{
Manish Pandya ${ }^{1, *}, J_{i t e n}$ Senta $^{2}$, Shrina Shah ${ }^{3}$, Ishita Mehta ${ }^{4}$ \\ ${ }^{1} \mathrm{HOD},{ }^{2,3,4}$ Resident, Dept. of Obstetrics \& Gynecology, Scientific Research Institute, Surendranagar, Gujarat, India \\ *Corresponding Author: Manish Pandya \\ Email: drmanish.pandya@gmail.com
}

Received: $7^{\text {th }}$ November, 2018

Accepted: $1^{\text {st }}$ December, 2018

\begin{abstract}
Congenital left ventricular aneurysm is a rare but potentially lethal condition. We describe a case of isolated congenital left ventricular aneurysm diagnosed at 28 weeks of gestation. In addition to standard imaging, we utilized color-coded transthoracic tissue Doppler techniques to further evaluate the aneurysm postnatally.
\end{abstract}

Keywords: Fetal echocardiography, Ventricular aneurysm, Congenital heart disease, Prenatal ultrasound.

\section{Introduction}

We would like to report the case of a left ventricular aneurysm diagnosed at 28 weeks of gestation. The diagnosis was eventually confirmed on postnatal echocardiography shows that severe left ventricular dysfunction. LVEF-10-15\% (Fig. 2).

\section{Case Report}

A 25-years old woman Primi para was referred for an abnormal cardiac finding on a comprehensive obstetric ultrasound performed at 28 weeks gestation. Due to late prenatal care, this was the patient's first ultrasound in the pregnancy. The left ventricle of the fetus was described as having an hourglass-like shape, and there was concern for irregular contractility. No other fetal abnormalities were identified, and there was no evidence of fetal hydrops. The patient had no significant past medical history and denied exposure to teratogens drug during the gestational assigned as per LMP.

Usg findings: single, live, intrauterine, cephalic presentation, Placenta- posterior, liquor adequate. Fetal heart sound-144BPM, Estimated fetal weight according to BPD, HC, AC, FL-1498+/-149.8 gram. The left ventricle of the fetus was described as having an hourglass-like shape, and there was concern for irregular contractility. So we advised for fetal echo for expert opinion.

Experts finding for fetal echo are left ventricular apex aneurysm, pericardial effusion, cardiomegaly, septum primum ASD, (partially AVSD) (shows Fig. 1) and on fetal Doppler scan report we found that MCA and ductus venosus normal, increase resistance umbilical artery. Advised karyotyping. (Patient refused)

Councelling done by our team regarding guraded prognosis and outcome, after knowing the guarded prognosis patient choose to deliver child at term.
Baby born $1^{\text {st }}$ November 2017. Baby admitted to NICU for further evaluation. Echo done on 1st day of life shows severe left ventricular dysfunction. LVEF$15 \%$ and patient put on digoxin, furosemide and other supportive treatment and further repeated echo done at 1 month interval and find out no progress.

Patient condition worsen on 27 march 2018 tachypnea was present, baby on oxygen support with nasal prong, dobutamine and other supportive care started and blood investigation shows $\mathrm{Hb}-9.6 \%$, Tc20140, Dc-486000, CRP- 24, $\mathrm{Na}^{+}-132, \mathrm{~K}^{+}-6.35$, Creatinin -0.35 and LVEF decrease 10 to $15 \%$ (shows Fig. 2). And patient shifted to intensive care unit and died on 4 months 29 days of life. 


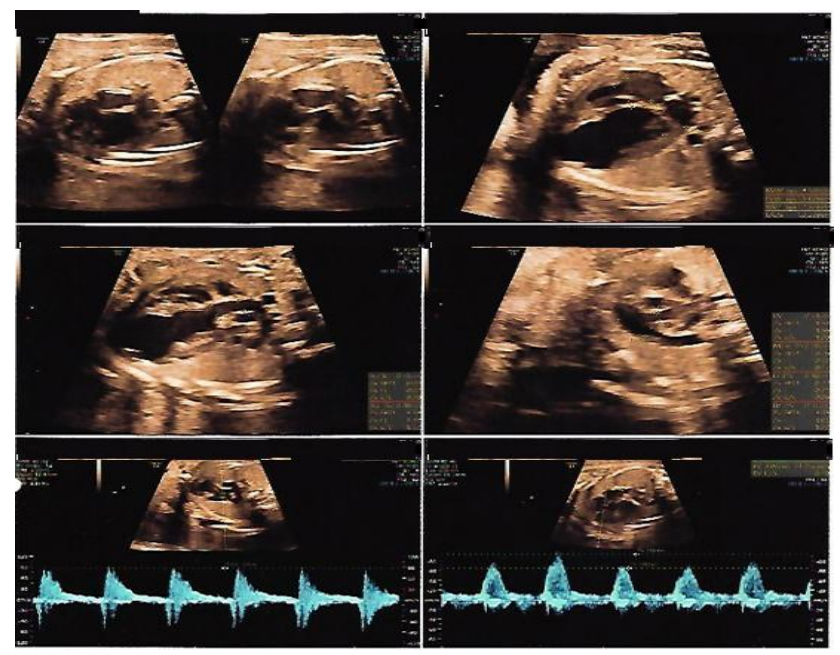

Fig. 1: Left ventricular apex aneurysm, pericardial effusion, cardiomegaly, septum primum ASD

\section{Fig. 2: Postnat echo findings}

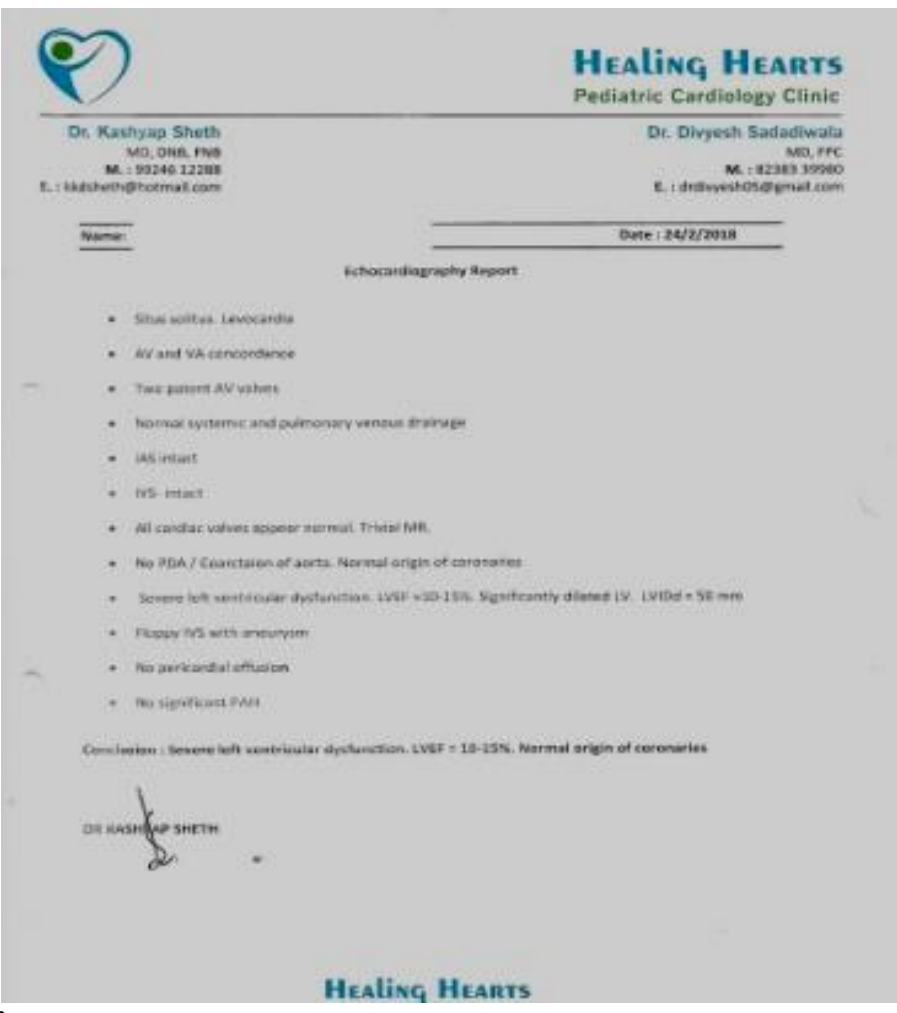

\section{Discussion}

Congenital ventricular aneurysm is a rare condition characterized by protrusion or out-pouching of the ventricular wall, especially of the left ventricle. ${ }^{1}$ The advantage of ultrasound and echocardiography has led to earlier detection of congenital ventricular aneurysm. It is appear to be due to developmental anomalies involving the left ventricular myocardium, thought to result from a focal weakening of the ventricular wall. Fetal viral infection and myocardial ischemia have been considered possible etiologies. ${ }^{2}$ congenital ventricular aneurysm is estimated to occur in 0.5 per 100,000 live births. ${ }^{3}$ Although the distinction between ventricular aneurysm and ventricular diverticulum remains inconsistent, it is important to distinguish the two entities, because the treatment and prognosis can differ. The classification criteria include connection to the ventricular cavity, wall composition, wall motion, and association with intracardiac abnormalities or midline defects. ${ }^{4}$ Ventricular aneurysm is a recent addition to the list of diseases that can be diagnosed before birth; the first case was reported in $1990 .{ }^{3}$ Classically, the USG diagnosis of a ventricular aneurysm is made based on the presence of a wide-mouthed ventricular outpouching along with the presence of paradoxical systolic expansion, though this may not always be seen. The aneurysm may arise from the ventricular septum, 
the wall, or the apical region. This fetus showed no other intra- or extracardiac anomaly; other anomalies are only rarely associated with such aneurysms. ${ }^{5}$ The etiology is unknown, but Surgical correction has been confined to symptomatic cases, while conservative treatment is used for asymptomatic cases. The prognosis varies, ranging from no symptoms to heart failure. ${ }^{6}$ The optimal treatment of congenital ventricular diverticula and congenital ventricular aneurysms remains unclear. When symptomatic, or when associated with other cardiac abnormalities, surgical treatment is usually recommended. ${ }^{7}$ The prognosis of a fetal left ventricular aneurysm depends on the time of detection, the size and the progression of the aneurysm, the presence or absence of compression of the fetal lungs, involvement of the mitral opening, reversal of atrial shunt, associated cardiac failure, and the presence of myocardial damage or connective tissue disorders. ${ }^{8,9}$ During routine second-trimester ultrasound examinations, the four-chamber view of the heart is essential to aid in the diagnosis of congenital heart disease. If an abnormality is detected, echocardiography is the standard modality to further evaluate the fetal heart.

\section{Conclusion}

A 25-year-old woman primi para was referred for an abnormal cardiac finding on a comprehensive obstetric ultrasound performed at 28 weeks' gestation. Due to late prenatal care, this was the patient's first ultrasound in the pregnancy. Fetal echo shows left ventricular apex aneurysm, pericardial effusion, cardiomegaly, septum primum ASD.

For early diagnosis of congenital anomalies it is advisable that fetal anomaly scan during 18-20 weeks of gestations. To diagnose congenital heart defects during antenatal period by ultrasound must see for 4 chamber view of heart with 3 vessels. If you see only 4 chamber view of heart than it has only $65-\%$ specificity while if 4 chamber \& 3 vessels view of heart gives you $81 \%$ specificity in early detection of congenital heart anamolies. ${ }^{10,11}$

\section{References}

1. Papagiannis J, Van Praagh R, Schwint O, D’Orsogna L, Qureshi F, Reynolds J, Kallfelz C, Nozar J. Congenital left ventricular aneurysm: clinical, imaging, pathologic, and surgical findings in seven new cases. Am Heart J. 2001;141:491-499.

2. Gembruch U, Steil E, Redel D A, Hansmann M. Prenatal diagnosis of a left ventricular aneurysm. Prenat Diagn. 1990;10:203-209.

3. Chew C, Halliday JL, Riley MM, Penny DJ. Populationbased study of antenatal detection of congenital heart disease by ultrasound examination. Ultrasound Obstet Gynecol. 2007;29:619-24.

4. Del Río M, Martínez J M, Bennasar M. Prenatal diagnosis of a right ventricular diverticulum complicated by pericardial effusion in the first trimester. Ultrasound Obstet Gynecol. 2005;25:409-411.
5. Gembruch U, Steil E, Redel DA, Hansmann M. Prenatal diagnosis of a left ventricular aneurysm. Prenat Diagn. 1990;10:203-9.

6. Sherman SJ, Leenhouts KH, Utter GO, Litaker M, Lawson P. Prenatal diagnosis of left ventricular aneurysm in the late second trimester: a case report. Ultrasound Obstet Gynecol. 1996;7:456-457.

7. Marijon E, Ou P, Fermont L. et al. Diagnosis and outcome in congenital ventricular diverticulum and aneurysm. $J$ Thorac Cardiovasc Surg. 2006;131:433-437.

8. Matias A, Fredouille C, Nesmann C, Azancot A. Prenatal diagnosis of left ventricular aneurysm: A report of three cases and a review. Cardiol Young. 1999;9:123-6.

9. Pipitone S, Sperandeo V, Mongiovi M, Roberto G, Centineo G. Prenatal diagnosis of ventricular aneurysm: A report of two cases and a review. Prenat Diagn. 2002;22:131-6.

10. Atlas of fetal echocardioghraphy $2^{\text {nd }}$ edition by Manish $r$ Pandya. (https://www.youtube.com/watch?v=ZiQvWe6fCZI\&t=16 $7 \mathrm{~s})$

11. Dr S. -J. Yoo Y.-H. Lee. Three-vessel view of the fetal upper mediastinum. (https://doi.org/10.1046/j.14690705.1997.09030173.x)

How to cite this article: Pandya M, Senta J, Shah S, Mehta I. A rare case report: Intrauterine diagnosis of fetal left ventricular aneurysm. Indian J Obstet Gynecol Res. 2018;5(4):579-581. 